Under the weaker hypothesis that $p x$ merely approaches a constant the same proof shows that $p w$ also approaches a constant.

A later note considers the existence and character of solutions of quasi-linear networks for periodic impressed force.

DePartment of TerRestrial MAgnetism,

Carnegie Institution of Washington

\title{
THE ELECTROSTATIC FIELD OF TWO COPLANAR PLATES
}

\section{EDMUND PINNEY}

1. Introduction. In a recent paper in the Philosophical Magazine $[1$, p. 168$],{ }^{1}$ N. Davy published what he called an "attempt" to obtain the electrostatic field about two thin, infinitely long, parallel, coplanar metallic plates of unequal width and at potentials $\pm V_{0}$. It was this remark, no doubt, that led M. C. Gray [2] to call this solution "tentative."

Actually, the solution given by Davy was but one of infinitely many that might fit his given conditions. The reason is that this is a two-dimensional potential problem, and in two-dimensional potential theory infinity is not a suitable zero point for the potential function as it is in three-dimensional potential theory. Consequently, to make the potential function definite, it is necessary either to specify its zero point or to specify some other condition which effectively does this. Davy made no such specification but chose the particular potential function which corresponds to the case in which the charges on the conductors are equal and opposite in sign.

It is the purpose of this paper to solve the problem fulfilling Davy's conditions, but in which the charge per unit length on one plate bears to the charge per unit length on the other plate a given ratio $r$.

For any $r \neq 1$, the charges on the plates may be increased until the potential difference between the two plates is $2 V_{0}$. If the zero point for the potential function is then taken as the point between the two plates at which the potential is the arithmetic mean of the potentials on the plates, one plate will be at potential $+V_{0}$ and the other will

Received by the editors May 20, 1946.

1 Numbers in brackets refer to the references cited at the end of the paper. 
be at potential $-V_{0}$, corresponding to Davy's given conditions. Davy's solution corresponds to the case $r=-1$.

2. The electrostatic field. Let the two plates have widths $h_{1}, h_{2}$, respectively, and let their adjacent edges be separated by a distance $h$. Define

$$
\begin{aligned}
k & =\left[h_{1} h_{2} /\left(h+h_{1}\right)\left(h+h_{2}\right)\right]^{1 / 2}, \quad k^{\prime}=\left(1-k^{2}\right)^{1 / 2}, \\
\sigma^{2} & =h_{1} /\left(h+h_{1}\right), \quad \sigma^{2}=h /\left(h+h_{1}\right)=1-\sigma^{2}, \\
\tau^{2} & =h_{2} /\left(h+h_{2}\right), \quad \rho^{2}=\left(h+h_{2}\right) /\left(h+h_{1}+h_{2}\right) .
\end{aligned}
$$

Let the plate of width $h_{1}$ occupy the interval $0<x<h_{1}$, and the plate of width $h_{2}$ occupy the interval $h+h_{1}<x<h+h_{1}+h_{2}$ on the $x$-axis of a rectangular $x, y$-coordinate system. Let $z=x+i y$, and let $E_{x}(z), E_{y}(z)$ denote the $x$ and $y$ components, respectively, of the electric field. Let the charge per unit length of the plate of width $h_{1}$ be $r$ times the charge per unit length on the plate of width $h_{2}$.

The method to be used here is that of $[3, \S 4]$. Let $x_{1}=0, x_{2}=h_{1}$, $x_{3}=h+h_{1}, x_{4}=h+h_{1}+h_{2}$. Take the "undisturbed" field to be zero, so that $f(x)=0$. Then by $[3, \S \S 4.1,4.7,4.9,4.10]$,

$$
\begin{aligned}
& E_{x}(z)-i E_{y}(z) \\
& \quad=(A z+B) /\left[z\left(z-h_{1}\right)\left(z-h-h_{1}\right)\left(z-h-h_{1}-h_{2}\right)\right]^{1 / 2},
\end{aligned}
$$

where the constants $A$ and $B$ must be determined.

3. Determination of the constants. By [4, pp. 24, 483],

$$
\int_{0}^{h_{1}} E_{y}(x+i 0) d x-r \int_{h+h_{1}}^{h+h_{1}+h_{2}} E_{y}(x+i 0) d x=0 .
$$

If the plate of width $h_{1}$ is to be at a potential $+V_{0}$, and the plate of width $h_{2}$ is to be at a potential $-V_{0}$, then

$$
\int_{h}^{h+h_{1}} E_{x}(x+i 0) d x=-2 V_{0} .
$$

By (3),

$$
\begin{aligned}
& E_{y}(x+i 0)=-(A x+B)\left[x\left(h_{1}-x\right)\left(h+h_{1}-x\right)\left(h+h_{1}+h_{2}-x\right)\right]^{-1 / 2}, 0 \\
& 0<x<h, \\
& E_{x}(x+i 0)=-(A x+B)\left[x\left(x-h_{1}\right)\left(h+h_{1}-x\right)\left(h+h_{1}+h_{2}-x\right)\right]^{-1 / 2}, \\
& h_{1}<x<h+h_{1},
\end{aligned}
$$


$E_{y}(x+i 0)=(A x+B)\left[x\left(x-h_{1}\right)\left(x-h-h_{1}\right)\left(h+h_{1}+h_{2}-x\right)\right]^{-1 / 2}$,

Therefore, from (4), (5), $h+h_{1}<x<h+h_{1}+h_{2}$.

$$
\left(P_{1}+r R_{1}\right) A+\left(P_{0}+r R_{0}\right) B=0, \quad Q_{1} A+Q_{0} B=V_{0}
$$

where

$$
\begin{aligned}
& \text { (7) } 2 P_{n}=\int_{0}^{h_{1}} x^{n}\left[x\left(h_{1}-x\right)\left(h+h_{1}-x\right)\left(h+h_{1}+h_{2}-x\right)\right]^{-1 / 2} d x, \\
& \text { (8) } 2 Q_{n}=\int_{h_{1}}^{h+h_{1}} x^{n}\left[x\left(x-h_{1}\right)\left(h+h_{1}-x\right)\left(h+h_{1}+h_{2}-x\right)\right]^{-1 / 2} d x, \\
& \text { (9) } 2 R_{n}=\int_{h+h_{1}}^{h+h_{1}+h_{2}} x^{n}\left[x\left(x-h_{1}\right)\left(x-h-h_{1}\right)\left(h+h_{1}+h_{2}-x\right)\right]^{-1 / 2} d x .
\end{aligned}
$$

From (6),

(10) $A=-V_{0}\left(P_{0}+r R_{0}\right) /(M+r N), B=V_{0}\left(P_{1}+r R_{1}\right) /(M+r N)$, where

$$
M=P_{1} Q_{0}-Q_{1} P_{0}, \quad N=R_{1} Q_{0}-Q_{1} R_{0} .
$$

$A$ and $B$ may be calculated from (10), (11) if $P_{0}, Q_{0}, R_{0}, P_{1}, Q_{1}, R_{1}$ are known. To determine them, in (7), (8), (9), respectively, make the substitutions

Then

$$
\begin{aligned}
& x=h+h_{1}-h /\left[1-\sigma^{2} \operatorname{sn}^{2}(\phi, k)\right], \\
& x=h_{1} /\left[1-\sigma^{2} \operatorname{sn}^{2}\left(\phi, k^{\prime}\right)\right], \\
& x=h_{1}+h /\left[1-\tau^{2} \operatorname{sn}^{2}(\phi, k)\right] .
\end{aligned}
$$

$$
\begin{aligned}
& P_{n}=k\left(h_{1} h_{2}\right)^{-1 / 2} \int_{0}^{K}\left\{h_{1}-h \sigma^{2} \operatorname{sn}^{2}(\phi, k) /\left[1-\sigma^{2} \operatorname{sn}^{2}(\phi, k)\right]\right\} n d \phi \\
& Q_{n}=k\left(h_{1} h_{2}\right)^{-1 / 2}
\end{aligned}
$$$$
\cdot \int_{0}^{K^{\prime}}\left\{h_{1}+h_{1} \sigma^{\prime 2} \operatorname{sn}^{2}\left(\phi, k^{\prime}\right) /\left[1-\sigma^{\prime 2} \operatorname{sn}^{2}\left(\phi, k^{\prime}\right)\right]\right\}^{n} d \phi,
$$

$$
\begin{aligned}
& R_{n}=k\left(h_{1} h_{2}\right)^{-1 / 2} \\
& \cdot \int_{0}^{K}\left\{h+h_{1}+h \tau^{2} \operatorname{sn}^{2}(\phi, k) /\left[1-\tau^{2} \operatorname{sn}^{2}(\phi, k)\right]\right\} n d \phi,
\end{aligned}
$$

where $K=K(k), K^{\prime}=K\left(k^{\prime}\right)$.

From (12)-(14),

$$
P_{0}=k\left(h_{1} h_{2}\right)^{-1 / 2} K, \quad Q_{0}=k\left(h_{1} h_{2}\right)^{-1 / 2} K^{\prime}, \quad R_{0}=k\left(h_{1} h_{2}\right)^{-1 / 2} K .
$$




$$
\begin{aligned}
& P_{1}=k\left(h_{1} h_{2}\right)^{-1 / 2}\left[h_{1} K-h F(\sigma, k)\right], \\
& Q_{1}=k\left(h_{1} h_{2}\right)^{-1 / 2}\left[h_{1} K^{\prime}+h_{1} F\left(\sigma^{\prime}, k^{\prime}\right)\right], \\
& R_{1}=k\left(h_{1} h_{2}\right)^{-1 / 2}\left[\left(h+h_{1}\right) K+h F(\tau, k)\right],
\end{aligned}
$$

where

$$
F(x, k)=\int_{0}^{K} \frac{x^{2} \operatorname{sn}^{2}(\phi, k)}{1-x^{2} \operatorname{sn}^{2}(\phi, k)} d \phi .
$$

Define the constant $a$ by

$$
\operatorname{sn}(a, k)=x / k .
$$

$a$ is real when $0<x<k$, but is complex when $k<x<1$. By $[5, \$ 22.74]$, $F(x, k)$ may be expressed in terms of the elliptic function of the third kind as

$$
F(x, k)=\operatorname{sc}(a, k) \text { nd }(a, k) \Pi(K, a, k) .
$$

By $[5, \S 22.74]$, this may be expresed in terms of theta and zeta functions as

$$
F(x, k)=\operatorname{sc}(a, k) \text { nd }(a, k)\left\{\frac{1}{2} \ln \frac{\Theta(K-a)}{\Theta(K+a)}+K Z(a)\right\} .
$$

Now the $\Theta$-function is even and periodic of period $2 K$, so

$$
F(x, k)=\operatorname{sc}(a, k) \text { nd }(a, k) K Z(a) .
$$

By $[5, \$ 22.731], F$ may be expressed in terms of the incomplete elliptic integral of the second kind as

$$
F(x, k)=\operatorname{sc}(a, k) \text { nd }(a, k)[K E(a, k)-a E] .
$$

By $[5, \S \$ 22.732,22.734]$,

$$
\begin{aligned}
E(c+i b, k)= & E(c, k)-i E\left(b, k^{\prime}\right)+\operatorname{isc}\left(b, k^{\prime}\right) \\
& \cdot\left[\operatorname{dn}\left(b, k^{\prime}\right)-k^{2} \operatorname{sn}(c, k) \operatorname{sn}(c+i b, k)\right]+i b .
\end{aligned}
$$

From (18) and $[5, \S \S 22.31,22.34,22.41]$,

$$
\begin{aligned}
& a=\operatorname{sn}^{-1}(x / k, k), \\
& 0<x<k \text {, } \\
& a=K+\operatorname{idn}^{-1}\left(k / x, k^{\prime}\right), \\
& k<x<1 \text {. }
\end{aligned}
$$

From (20), (21), and $[5, \S \S 22.11,22.31,22.34,22.41]$,

$$
E(a, k)=E\left(\mathrm{sn}^{-1}(x / k, k), k\right)
$$

when $0<x<k$, and 


$$
\begin{aligned}
E(a, k)= & E+i(1 / x)\left[\left(x^{2}-k^{2}\right)\left(1-x^{2}\right)\right]^{1 / 2} \\
& -i E\left(\operatorname{dn}^{-1}\left(k / x, k^{\prime}\right), k^{\prime}\right)+i \operatorname{dn}^{-1}\left(k / x, k^{\prime}\right)
\end{aligned}
$$

when $k<x<1$.

From (19), (21),

$$
\begin{array}{rlr}
F(x, k)= & x\left[\left(k^{2}-x^{2}\right)\left(1-x^{2}\right)\right]^{-1 / 2}\left[K E\left(\operatorname{sn}^{-1}(x / k, k), k\right)\right. \\
& \left.-E \operatorname{sn}^{-1}(x / k, k)\right], \\
F(x, k)= & -K+x\left[\left(x^{2}-k^{2}\right)\left(1-x^{2}\right)\right]^{-1 / 2}\left[K E\left(\operatorname{dn}^{-1}\left(k / x, k^{\prime}\right), k^{\prime}\right)\right. \\
& \left.+(E-K) \operatorname{dn}^{-1}\left(k / x, k^{\prime}\right)\right], & k<x<1 .
\end{array}
$$

From (16), (22),

$$
\begin{aligned}
P_{1}= & {\left[\left(h+h_{1}\right) /\left(h+h_{2}\right)\right]^{1 / 2} K-K E\left(\mathrm{dn}^{-1}\left(\tau, k^{\prime}\right), k^{\prime}\right) } \\
& +(K-E) \mathrm{dn}^{-1}\left(\tau, k^{\prime}\right), \\
Q_{1}= & k\left(h_{1} / h_{2}\right)^{1 / 2} K^{\prime}+K^{\prime} E\left(\mathrm{sn}^{-1}\left(\rho, k^{\prime}\right), k^{\prime}\right)-E^{\prime} \operatorname{sn}^{-1}\left(\rho, k^{\prime}\right), \\
R_{1}= & k\left(h_{1} / h_{2}\right)^{1 / 2} K+K E\left(\operatorname{dn}^{-1}\left(\sigma, k^{\prime}\right), k^{\prime}\right)-(K-E) \mathrm{dn}^{-1}\left(\sigma, k^{\prime}\right) .
\end{aligned}
$$

By (1), (2), $\rho=\sigma^{\prime} / k^{\prime}$. Therefore, by $[5, \S 22.11]$,

$$
\operatorname{dn}^{-1}\left(\sigma, k^{\prime}\right)=\operatorname{sn}^{-1}\left(\rho, k^{\prime}\right)
$$

By (11), (15), (23), (24),

$$
\begin{aligned}
N & =k\left(h_{1} h_{2}\right)^{-1 / 2}\left[K^{\prime} R_{1}-K Q_{1}\right] \\
& =k\left(h_{1} h_{2}\right)^{-1 / 2}\left[K E^{\prime}-K K^{\prime}+K^{\prime} E\right] \operatorname{sn}^{-1}\left(\rho, k^{\prime}\right) .
\end{aligned}
$$

By using Legendre's relation $[5, \$ 22.735]$,

$$
N=2^{-1} \pi k\left(h_{1} h_{2}\right)^{-1 / 2} \mathrm{sn}^{-1}\left(\rho, k^{\prime}\right) .
$$

By (1), (2), $\sigma \tau=k$. Therefore by $[5, \S 22.31]$ and (24),

$$
\operatorname{dn}^{-1}\left(\tau, k^{\prime}\right)=K^{\prime}-\operatorname{sn}^{-1}\left(\rho, k^{\prime}\right) \text {. }
$$

By $[5, \S 22.732]$,

$$
E\left(\mathrm{dn}^{-1}\left(\tau, k^{\prime}\right), k^{\prime}\right)=k h\left(h_{1} h_{2}\right)^{-1 / 2}-E\left(\operatorname{sn}^{-1}\left(\rho, k^{\prime}\right), k^{\prime}\right)+E^{\prime} .
$$

Substituting this into the expression for $P_{1}$ in (23), and using Legendre's relation, we obtain

$$
P_{1}=R_{1}-\pi / 2
$$

From (11), (15), (26),

$$
M=N-2^{-1} \pi Q_{0}
$$

From (15), (25), 


$$
M=-2^{-1} \pi k\left(h_{1} h_{2}\right)^{-1 / 2}\left[K^{\prime}-\mathrm{sn}^{-1}\left(\rho, k^{\prime}\right)\right] .
$$

From (10), (15), (23), (25), (27),

$$
\begin{aligned}
A= & (2 / \pi) V_{0}(1+r) K /\left[K^{\prime}-(1+r) \operatorname{sn}^{-1}\left(\rho, k^{\prime}\right)\right], \\
B= & V_{0}\left[\left(h+h_{1}\right)\left(h+h_{2}\right)\right]^{-1 / 2}\left\{1-2^{-1} \pi(1+r)\left[k\left(h_{1} / h_{2}\right)^{1 / 2} K\right.\right. \\
+ & K E\left(\operatorname{sn}^{-1}\left(\rho, k^{\prime}\right), k^{\prime}\right)-(K-E) \\
& \left.\left.\cdot \operatorname{sn}^{-1}\left(\rho, k^{\prime}\right)\right]\right\} /\left[K^{\prime}-(1+r) \operatorname{sn}^{-1}\left(\rho, k^{\prime}\right)\right] .
\end{aligned}
$$

The constants in (28), (29) are given in (1), (2). $K$ and $E$ are the complete elliptic integrals of the first and second kinds, respectively, with respect to the modulus $k$, and $K^{\prime}$ is the complete elliptic integral of the first kind with respect to the modulus $k^{\prime}$.

\section{REFERENCES}

1. N. Davy, Philosophical Magazine (7) vol. 36 (1945) pp. 153-169.

2. M. C. Gray, Mathematical Reviews vol. 7 (1946) p. 179.

3. E. Pinney, Ann. of Math. (2) vol. 47 (1946).

4. Stratton, Electromagnetic theory, McGraw-Hill, 1941.

5. E. T. Whittaker and G. N. Watson, Modern analysis, Cambridge University Press, 1935.

Oregon State College 